\title{
Prix de la coopération belge au développement 2010, 24 mars 2010
}

\section{Biodiversité et environnement pour une vie meilleure}

Dans le cadre de la rre édition du Prix de la Coopération belge au développement, qui a pour thème "Biodiversité et environnement pour une meilleure vie ", a eu lieu ce mercredi 24 mars, au Palais des colonies du Musée royal de l'Afrique centrale (MRAC), à Tervuren, une conférence suivie de la remise des prix.

Le Prix de la Coopération au Développement a été créé en 1998 par la Coopération belge au Développement. Il a pour but d'encourager des jeunes du Nord et du Sud à s'intéresser à la coopération internationale, et à y rester engagés lorsqu'ils auront terminé leurs études. Dès l'origine, le MRAC a été chargé de son organisation et de sa coordination.

Depuis 2009, le Prix de la Coopération au développement, devenu « Prix de la Coopération belge au développement ", est chaque fois organisé autour d'un thème. Le thème de l'édition 2010 est en relation directe avec l'année internationale de la biodiversité.

Tous les deux ans, 3 prix sont attribués sous forme de subsides pour la participation à des conférences, stages, formations ou l'achat d'équipement scientifique :

- une ligne de crédit d'une valeur de 5000 euros pour un étudiant rattaché à une université belge ;

- une ligne de crédit d'une valeur de 5000 euros pour un étudiant rattach une université d'un pays partenaire de la Coopération belge au développement ;

- une ligne de crédit d'une valeur de 15000 euros pour un jeune chercheur rattaché à une université d'un pays partenaire de la Coopération belge au développement.

Le Prix couronne des travaux scientifiques d'étudiants (thèse de licence ou mâ̂trise) et de jeunes chercheurs (thèse de doctorat ou publication) qui contribuent fortement à la connaissance dont le Sud pourra bénéficier pour son développement. Le développement durable et la lutte contre la pauvreté sont prioritaires. Les candidats sont des ressortissants de Belgique et de pays partenaires de la coopération belge universitaire et gouvernementale au développement. La sélection des candidatures s'effectue par un jury composé de représentants d'universités et d'instituts scientifiques belges. Le jury est présidé par le directeur du MRAC, M. Guido Gryseels. 
Dans le cadre de l'édition 2010 du Prix de la Coopération belge au développement, organisé sur le thème "Biodiversité et environnement pour une meilleure vie ", les travaux de 4 belges et 6 étudiants et chercheurs du Sud ont été nominés parmi un grand nombre de candidatures. Ces derniers ont été invités en Belgique à cette occasion. Les candidats retenus ont pu présenter leur travail oralement aux membres du jury. Celui-ci a ensuite sélectionné un lauréat pour chacune des trois catégories.

Pendant la journée thématique, les chercheurs nominés ont présenté leur travail à un public plus large, alternant avec des présentations réalisées par des experts, dans le domained'environnementet de biodiversité. La journée thématique a étésuivie par une session de présentation de posters, au cours de laquelle les nominés ont pu commenter leurs travaux, qui ont par ailleurs été décrits brièvement lors de la remise des prix qui clôturait la soirée.

Puis, en présence de $M$. François Bontemps, directeur général adjoint de la Coopération au développement, le président du jury, $M$. Guido Gryseels, a proclamé les 3 lauréats.

Les lauréats 2010 sont :

- dans la catégorie des étudiants liés à une université belge : Sarah HAESAERT, qui s'est plongée dans l'ethnobotanique appliquée, et plus particulièrement l'identification, l'utilisation et l'importance socio-économique des plantes sauvages comestibles chez les Turumbu (DRC) : " Toegepaste etnobotanie : identificatie, gebruik en socio-economisch belang van wilde eetbare planten bij de Turumbu (DR Congo, District Tshopo ) ", prom. : prof. dr. ir. Patrick Van Damme et ir. Céline Termote, UGent.

- dans la catégorie des étudiants liés à une université d'un pays partenaire : Joseph MACHARIA, un étudiant du Kenya, qui s'est concentré sur la conservation de la forêt et la création de revenus à travers d'une étude du cas de la forêt de Kakamega : "Status and the potential of stingless bees (Apidae : Meliponinae) for forest conservation and income generation : case study of Kakamega forest"

- dans la catégorie des jeunes chercheurs d'un pays en développement lié à une université/institution de recherche d'un pays partenaire : Mohamed Omar Said MOHAMED, un jeune chercheur du Kenya, s'est consacré aux effets de la pollution par eaux usées et de l'exploitation du bois sur la structure et le développement des mangroves de Mobasa (Kenya) : " Are peri-urban mangrove forests viable? Effects of sewage pollution and wood exploitation on the structure and development of the mangroves of Mombasa (Kenya)".

Une brève description des travaux couronnés et nominés est disponible via http ://devcoprize.africamuseum.be. 